DOI: $10.1007 / \mathrm{s} 10357-009-1737-8$

\section{Übermittlung von Umweltinformationen; Verweis auf Bundesrecht}

UIG §3 Abs. 2, §9 Abs. 2; LUIG BW §3 Abs. 1; VwGO §137 Abs. 1

Hat der Landesgesetzgeber davon abgesehen, eine eigene Vollregelung des Umweltinformationsrechts zu treffen, sondern in seinem Landesumweltinformationsgesetz die entsprechende Geltung bestimmter Vorschriften des Umweltinformationsgesetzes des Bundes angeordnet, finden diese Vorschriften nicht als Bundesrecht, sondern als irrevisibles Landesrecht Anwendung.

BVerw G, Beschluss vom 2. 7.2009- 7 B 9.09 -

VGH Mannheim vom 25.11.2008 - 10 S 2702/06, (in diesem Heft)

[1] Der Kläger begehrt von der beklagten Landesanstalt Umweltinformationen. Das Verwaltungsgericht hat die Beklagte antragsgemäß verpflichtet, dem Kläger die begehrten Informationen in Form einer Datei zu übermitteln. Der Verwaltungsgerichtshof hat die Berufung der Beklagten zurückgewiesen: Der Kläger habe gemäß $\$ 3$ Abs. 1 des Landesumweltinformationsgesetzes (LUIG) vom 7.3.2006 (GBl. S. 50) in Verbindung mit $\$ 3$ Abs. 2 Satz 2 des Umweltinformationsgesetzes des Bundes (UIG) vom 22.12.2004 (BGBl. I S. 3704) einen Anspruch darauf, dass ihm die erbetenen Informationen in Dateiform übermittelt würden. Der Anspruch auf Zugang zu den erbetenen Informationen sei nicht gemäß $₫ 3$ Abs. 1 LUIG i.V.m. $\$ 9$ Abs. 2 UIG ausgeschlossen, weil private Dritte die begehrten Umweltinformationen der Beklagten übermittelt hätten und eine Offenbarung der Informationen nachteilige Auswirkungen auf die Interessen der Dritten hätte.

\section{Aus den Gründen:}

[3] Die Beschwerde ist unbegründet. Die Rechtssache hat nicht die allein geltend gemachte grundsätzliche Bedeutung im Sinne des $\$ 132$ Abs. 2 Nr. 1 VwGO.

[4] Die Beklagte möchte Fragen geklärt wissen, die sich zur Auslegung und Anwendung des $₫ 3$ Abs. 2 Satz 2 UIG und des $\$ 9$ Abs. 2 UIG stellen. Die hierzu aufgeworfenen Fragen rechtfertigen die Zulassung der Revision nicht Sie sind in dem angestrebten Revisionsverfahren nicht klärungsfähig. Die Antwort auf sie richtet sich nach irrevisiblem Landesrecht, an dessen Auslegung durch den Verwaltungsgerichtshof das Bundesverwaltungsgericht im Revisionsverfahren gebunden wäre.

[5] Das Umweltinformationsgesetz des Bundes gilt nur für die informationspflichtigen Stellen des Bundes. Der Landesgesetzgeber in Baden-Württemberg hat davon abgesehen, für die informationspflichtigen Stellen des Landes, $\mathrm{zu}$ denen die beklagte Landesanstalt gehört, eine eigene Vollregelung des Umweltinformationsrechts zu treffen. Er hat vielmehr in $\$ 3$ Abs. 1 LUIG für den Zugang zu und die Verbreitung von Umweltinformationen die entsprechende Geltung bestimmter Vorschriften des Umweltinformationsgesetzes des Bundes angeordnet.

[6] Die Vorschriften des Umweltinformationsgesetzes kommen hier deshalb nicht aufgrund bundesrechtlicher Anordnung, sondern allein deswegen zur Anwendung, weil Landesrecht hierauf verweist ( $\$ 3$ LUIG). Diese landesrechtliche Verweisung ist für die Geltung des Umweltinformationsgesetzes sowohl dem Grunde als auch dem Umfang nach maßgeblich. Ist aber eine Vorschrift des Bundesrechts ausschließlich kraft eines Gesetzesbefehls des Landesgesetzgebers anzuwenden, handelt es sich insoweit um irrevisibles Landesrecht. Irrevisibles Landesrecht liegt vor, wenn eine Vorschrift des Bundesrechts nicht kraft Gesetzesbefehls des
Bundesgesetzgebers, sondern nur kraft der Bezugnahme im Landesrecht und damit aufgrund einer gesetzgeberischen Entscheidung des Landes Geltung beansprucht (Beschlüsse vom 7.6.2002 - 9 B 30.02, juris; und vom 10.8.2007 - 9 B 19.07, Buchholz $310 \$ 137$ Abs. 1 VwGO Nr. 29).

...

\section{Bedeutungslosigkeit eines Verfahrensfehlers für Landschaftsschutzgebietsverordnung; Voraussetzungen für eine Unterschutzstellung}

LNatG M-V §23 Abs. 1, §30 Abs. 4, §31 Abs. 2 und 7

1. Eines Erörterungstermines oder der anderweitigen Ergebnismitteilung gemäß $₫ 30$ Abs. 4 LNatG M-V bedarf es lediglich bezüglich ,fristgerecht" vorgebrachter Bedenken und Anregungen.

2. Daraus, dass ein Verfahrensfehler nicht bereits nach $₫ 31$ LNatG M-V unbeachtlich ist, folgt im Einzelfall nicht zwingend, dass eine Landschaftsschutzgebietsverordnung wegen eines Verstoßes gegen $\mathbb{\$} 30$ Abs. 4 LNatG M-V unwirksam wäre.

3. Da die Funktion der Ergebnismitteilung nach $₫ 30$ Abs. 4 LNatG M-V lediglich darin besteht, die Präklusionsfrist des $₫ 31$ Abs. 2 LNatG M-V auszulösen, also im öffentlichen Interesse eine für die Adressaten der Ergebnismitteilung negative Rechtsfolge nach sich ziehen soll, führt der Verstoß gegen $\$ 30$ Abs. 4 LNatG M-V durch Unterlassen der Mitteilung zu keinen für sie nachteiligen Konsequenzen. Angesichts dieses Befundes hat der in Rede stehende Verfahrensfehler gegenüber den Beteiligten bzw. Betroffenen kein bzw. allenfalls geringes Gewicht, das es nicht rechtfertigen kann, an ihn die Rechtsfolge der Unwirksamkeit der Verordnung zu knüpfen bzw. anzunehmen, dass der Gesetzgeber dies wollte.

4. In Anerkennung des Anliegens der Normerhaltung kann im Einzelfall ausnahmsweise die Verletzung von Beteiligungsrechten im Normsetzungsverfahren dann nicht zur Unwirksamkeit der angegriffenen Norm führen, wenn ausgeschlossen werden kann, dass die Norm ohne den Verfahrensfehler einen anderen Inhalt erhalten hätte.

5. Der Rückgriff auf eine Übersichtskarte ist im Rahmen des $₫ 30$ Abs. 7 Satz 1 Nr. 2, 1. Halbsatz LNatG $\mathrm{M}-\mathrm{V}$ zulässig.

6. Es ist nach $₫ 23$ Abs. 1 LNatG M-V nicht erforderlich, dass das Schutzgebiet im Vergleich zu anderen Gebieten im Land schutzwürdiger oder schutzbedürftiger erscheint. Liegen die gesetzlichen Voraussetzungen für die Unterschutzstellung vor, ist diese zulässig, ohne dass es darauf ankäme, wie sich die Verhältnisse anderen Ortes darstellen.

7. Im Rahmen seines naturschutzrechtlichen „Normsetzungsermessens" hat der Verordnungsgeber auch verfassungsrechtlich geschützte Positionen, wie etwa die gemeindliche Planungshoheit bzw. die Garantie der kommunalen Selbstverwaltung und die privaten Eigentümerbelange, zu berücksichtigen.

8. Konkretisieren sich die planerischen Zielsetzungen einer Gemeinde erst nach dem Zeitpunkt der einstweiligen Sicherstellung, unterliegen diese Planungen ihrem naturschutzrechtlichen Regime.

OVG Greifswald, Urteil vom 14. 10.2008 - 4 K 25/06- 